\title{
Anomalous Diffusion Profiles of Ag in CdTe due to Chemical Self-Diffusion
}

\author{
H. Wolf, ${ }^{1}$ F. Wagner, ${ }^{1}$ Th. Wichert, ${ }^{1}$ and Isolde Collaboration ${ }^{2}$ \\ ${ }^{1}$ Technische Physik, Universität des Saarlandes, D-66041 Saarbrücken, Germany \\ ${ }^{2}$ CERN, EP Division, CH-1211 Geneve 23, Switzerland \\ (Received 22 September 2004; published 1 April 2005)
}

\begin{abstract}
Anomalous diffusion profiles of $\mathrm{Ag}$ in single crystalline CdTe were observed using the radiotracer ${ }^{111} \mathrm{Ag}$. The diffusion anneals were performed at $800 \mathrm{~K}$ under $\mathrm{Cd}$ or Te vapor and in a vacuum for different Ag concentrations. The measured Ag profiles directly reflect the distribution of the self-interstitials and vacancies of the $\mathrm{Cd}$ sublattice and are the result of chemical self-diffusion which describes the variation of the deviation from stoichiometry of the binary crystal as a function of depth and time.
\end{abstract}

Understanding and control of diffusion profiles of intrinsic and extrinsic defects in semiconductors is of central importance for developing electronic and optoelectronic devices with high integration and reduced structural sizes. At temperatures required for crystal growth and device processing, intrinsic point defects, like vacancies and self-interstitials, are highly mobile and can interact with extrinsic point defects, like dopant atoms and impurities. For this reason, detailed studies of the mobility and interaction of various defects were performed in elemental semiconductors, like $\mathrm{Si}$ and $\mathrm{Ge}$, and, but to a lesser extent, in various compound semiconductors [1]. The various shapes of the experimentally observed diffusion profiles deliver valuable information about different diffusion mechanisms, like, e.g., in the case of $\mathrm{Au}$ in $\mathrm{Si}$ and $\mathrm{Cu}$ in Ge [1]. Common to all diffusion profiles reported so far is their monotonously decreasing depth profile if the source is located at the surface of the crystal and the diffusion length is small compared with the thickness of the crystal [2]. In contrast to $\mathrm{Si}$ and $\mathrm{Ge}$, in compound semiconductors large concentrations of intrinsic point defects as compared to thermally generated defects can be obtained by inducing slight deviations from stoichiometry in the respective material. Thus, by exposing a compound semiconductor to the vapor pressures of one of its constituents, variations of the deviation from stoichiometry of the binary crystal as a function of depth and time are generated and, thereby, concentration gradients of intrinsic point defects.

Up to now, this possibility for controlling diffusion profiles of extrinsic defects does not seem to have been studied. Here, as a model system, the diffusion of the group I element $\mathrm{Ag}$ was investigated in the binary compound semiconductor $\mathrm{CdTe}$. The group $\mathrm{Ib}$ elements $\mathrm{Cu}$, $\mathrm{Ag}$, and $\mathrm{Au}$ belong to the important impurities in II-VI compound semiconductors [3,4]: Besides substitutional lattice sites, they are reported to occupy interstitial lattice sites, thereby acting as amphoteric dopants and already exhibiting a high diffusivity at relatively low temperatures. It will be shown that due to the interaction of extrinsic defects with intrinsic point defects anomalous diffusion profiles are created that can be varied between peak shaped and $\mathrm{U}$ shaped depending on the diffusion conditions. Preliminary results of this work were published in [5-7].

The diffusion of Ag in CdTe was investigated using the radiotracer ${ }^{111} \mathrm{Ag}$ which decays to stable ${ }^{111} \mathrm{Cd}$ with a halflife of $7.45 \mathrm{~d}$. The radiotracer was implanted into one side (called "front") of polished and etched single crystals of 0.5 or $0.8 \mathrm{~mm}$ thickness (CRYSTEC $\mathrm{GmbH}$, Berlin, Germany, and R. Grill, Charles University, Prague, Czech Republic) [6]. The implantation energy was 60 or $80 \mathrm{keV}$ yielding a Gaussian shaped ${ }^{111} \mathrm{Ag}$ profile, located at a depth of about $30 \mathrm{~nm}$ (width about $20 \mathrm{~nm}$ ). The dose of implanted ${ }^{111} \mathrm{Ag}$ was in the range $10^{11}$ to $10^{12} \mathrm{~cm}^{-2}$. In order to increase the concentration of $\mathrm{Ag}$ atoms a film of stable $\mathrm{Ag}$ was evaporated onto the front side before diffusion. For diffusion, the crystals were enclosed in an evacuated quartz ampoule. By adding metallic $\mathrm{Cd}$ or $\mathrm{Te}$ the corresponding vapor pressure was provided for changing the stoichiometry of the crystal. The ${ }^{111} \mathrm{Ag}$ concentration depth profile was determined by mechanical polishing of the crystal and recording the intensity of the $342 \mathrm{keV} \gamma$ line of the radioactive decay of ${ }^{111} \mathrm{Ag}$ [8]; the thicknesses of the abraded layers ranged between 1 and $40 \mu \mathrm{m}$.

The data in Fig. 1 (closed circles) show concentration profiles of ${ }^{111} \mathrm{Ag}$ in CdTe after annealing at three different temperatures in vacuum. The monotonously decreasing concentration profile after annealing at $570 \mathrm{~K}$ for $30 \mathrm{~min}$ and the almost constant profile obtained after annealing at $670 \mathrm{~K}$ (60 min) are in qualitative agreement with extrapolated diffusion data reported for the temperature range $320-345 \mathrm{~K}$ [9]. After annealing at $800 \mathrm{~K}$ (60 min), instead of the flat profile observed at $670 \mathrm{~K}$ two depletion layers of about $100 \mu \mathrm{m}$ width appear at both surfaces of the crystal. The Ag concentration at the surface is reduced by 1 order of magnitude as compared to the interior of the crystal.

The diffusion properties observed at $800 \mathrm{~K}$ [Fig. 1(c)] are investigated in more detail and the results are shown in Fig. 2 (left panels). Now, well defined external conditions are used during the diffusion anneal at $800 \mathrm{~K}(60 \mathrm{~min})$. After diffusion under $\mathrm{Cd}$ vapor, Fig. 2(a) shows a symmetrical, peak-shaped Ag profile located at the center of the crystal and a strongly depleted region of about $250 \mu \mathrm{m}$ 


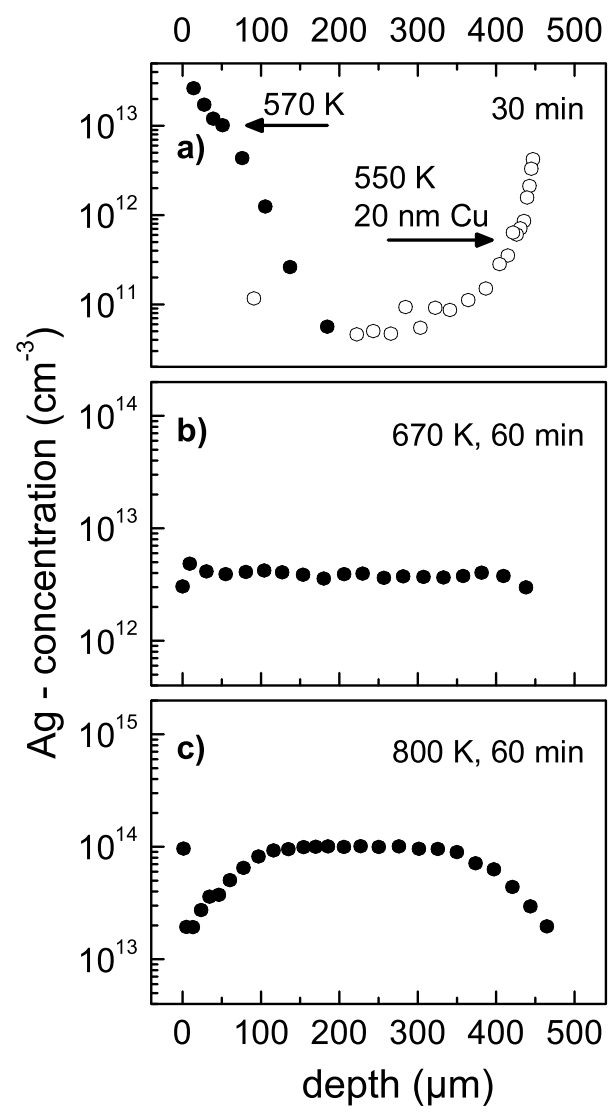

FIG. 1. Concentration profiles of ${ }^{111} \mathrm{Ag}$ in CdTe after implantation into the front side of a CdTe crystal and diffusion anneals under vacuum at different temperatures and for the times indicated (closed circles). In addition, the top panel shows a ${ }^{111} \mathrm{Ag}$ profile observed after codiffusion of $\mathrm{Cu}$, deposited as $20 \mathrm{~nm}$ thick film, at $550 \mathrm{~K}$ (open circles).

towards both surfaces of the $800 \mu \mathrm{m}$ thick crystal. In contrast, diffusion under Te pressure [Fig. 2(b)] causes layers of about $20 \mu \mathrm{m}$ below each surface, containing high concentrations of Ag atoms, whereas the Ag concentration in the interior of the crystal is significantly lower. The asymmetry of the profile excludes that a significant portion of the $\mathrm{Ag}$ atoms reached the backside of the crystal from the front side via the external vapor phase. In a second series, the $\mathrm{Ag}$ concentration was increased by evaporation of a $30 \mathrm{~nm}$ layer of stable $\mathrm{Ag}$ on the front side of the sample. The data in Figs. 2(c) and 2(d) show that increasing the $\mathrm{Ag}$ concentration from about $10^{13}$ to $10^{18} \mathrm{~cm}^{-3}$ yields qualitatively the same profiles as visible in the corresponding left panels of Fig. 2, whereby in Fig. 2(c) the peak-shaped profile is broader and a significant segregation of $\mathrm{Ag}$ atoms at both surfaces seems to occur. It is excluded that the depleted regions, observable in Figs. 1(c), 2(a), and 2(c), are caused by out diffusion of ${ }^{111} \mathrm{Ag}$ atoms for the loss of radioactive atoms was below $5 \%$ in all experiments.

In summarizing the results so far, it can be stated that the data in Fig. 2 show a drastic dependence of the ${ }^{111} \mathrm{Ag}$

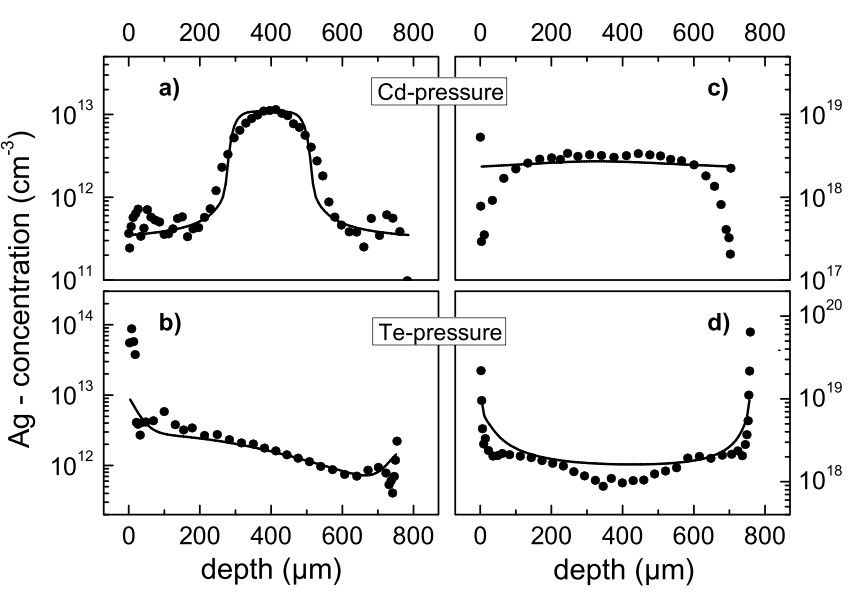

FIG. 2. Influence of external conditions on the concentration profiles of ${ }^{111} \mathrm{Ag}$ in CdTe observed after diffusion at $800 \mathrm{~K}$ (60 min) at (a), (b) low and at (c), (d) high Ag concentrations. The solid lines are fits to the experimental data as described in the text.

profiles on the external partial pressures of $\mathrm{Cd}$ and $\mathrm{Te}$ which alter the stoichiometry of the crystals during annealing. Further, the comparison of the profiles in Figs. 2(a) and 3 yields that the CdTe crystals used for the present experiments were Te rich: The only difference between both experiments is the preannealing of the CdTe crystal in Fig. 3 under Cd vapor at $800 \mathrm{~K}$ for $24 \mathrm{~h}$. From the data in Fig. 3 it is obvious that the subsequent diffusion anneal under $\mathrm{Cd}$ vapor does not lead to the "snow plow" effect that is visible in Fig. 2(a). This snow plow effect is inhibited in Fig. 3 for the crystal is $\mathrm{Cd}$ saturated by the preannealing to such an extent that no significant gradient in the deviation from stoichiometry can be generated by the subsequent diffusion annealing for $60 \mathrm{~min}$. In contrast, in the Te-rich crystal used for the experiment in Fig. 2(a), the external $\mathrm{Cd}$ vapor introduces mobile Cd self-interstitials $\left(\mathrm{Cd}_{\mathrm{i}}\right)$ from both surfaces which are able to introduce concentration gradients of the intrinsic defects. In this

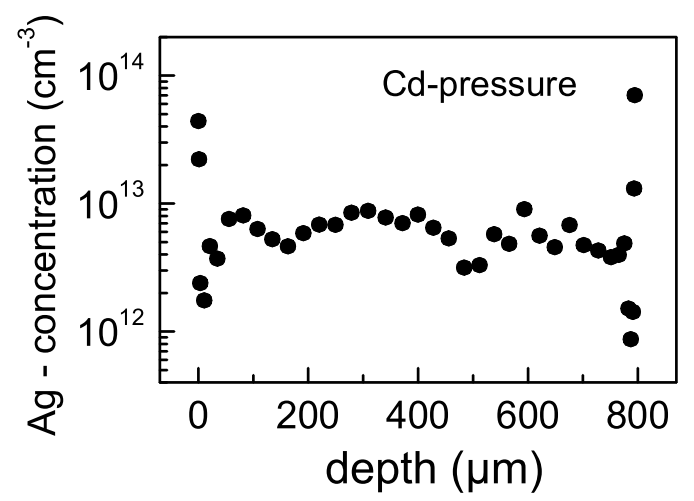

FIG. 3. Ag profile after diffusion under $\mathrm{Cd}$ pressure at $800 \mathrm{~K}$ (60 $\mathrm{min})$ in a CdTe crystal that was preannealed under $\mathrm{Cd}$ pressure at $800 \mathrm{~K}$ for $24 \mathrm{~h}$. 
case, according to the reaction

$$
\mathrm{Ag}_{\mathrm{s}}+\mathrm{Cd}_{\mathrm{i}} \rightleftharpoons \mathrm{Ag}_{\mathrm{i}}
$$

$\mathrm{Cd}_{\mathrm{i}}$ atoms produce mobile $\mathrm{Ag}$ interstitials $\left(\mathrm{Ag}_{\mathrm{i}}\right)$ via a replacement of substitutional $\mathrm{Ag}$ atoms $\left(\mathrm{Ag}_{\mathrm{s}}\right)$. In order to warrant that the snow plow effect caused by the $\mathrm{Cd}_{\mathrm{i}}$ defects works these defects should be less mobile than $\mathrm{Ag}_{\mathrm{i}}$ atoms so that a recombination of the $\mathrm{Ag}_{\mathrm{i}}$ atoms with the remaining $\mathrm{Cd}$ vacancies $\left(V_{\mathrm{Cd}}\right)$ in the interior of the crystal ahead of the $\mathrm{Cd}_{\mathrm{i}}$ defects is possible according to

$$
\mathrm{Ag}_{\mathrm{i}}+V_{\mathrm{Cd}} \rightleftharpoons \mathrm{Ag}_{\mathrm{s}}
$$

That the snow plow effect leading to the peak-shaped profile in Fig. 2(a) is effected by $\mathrm{Cd}_{\mathrm{i}}$ defects and not by Te vacancies or $\mathrm{Cd}$ antisite defects, which both, in principle, might be generated by the $\mathrm{Cd}$ pressure, too, is shown by the second profile (open circles) in Fig. 1(a): This profile also exhibits a snow plow effect in pushing the ${ }^{111} \mathrm{Ag}$ atoms to the back side of the CdTe crystal. This ${ }^{111} \mathrm{Ag}$ profile is observable after a diffusion anneal at $550 \mathrm{~K}$ (30 min) if a $\mathrm{Cu}$ layer was deposited on the front side of the CdTe crystal following implantation of the ${ }^{111} \mathrm{Ag}$ atoms [6]. Now, mobile interstitial $\mathrm{Cu}$ atoms $\left(\mathrm{Cu}_{\mathrm{i}}\right)$ substitute the $\mathrm{Cd}_{\mathrm{i}}$ defects in Eq. (1) which push the generated $\mathrm{Ag}_{\mathrm{i}}$ atoms from the front side to the back side of the CdTe crystal in contrast to the diffusion anneal without $\mathrm{Cu}$ [see closed circles in Fig. 1(a)]. Since the $\mathrm{Cu}_{\mathrm{i}}$ defects were generated only at the front side of the crystal, the $\mathrm{Ag}$ profile is asymmetric, in this case.

On the basis of the data present here a quantitative model for describing the different diffusion profiles is proposed. For the sake of simplicity, it is assumed that (i) the Te sublattice is perfect and not affected by the different thermal treatments in a significant way, (ii) that the Ag atoms are only incorporated as either $\mathrm{Ag}_{\mathrm{s}}$ on $\mathrm{Cd}$ sites or $\mathrm{Ag}_{\mathrm{i}}$, on interstitial sites, i.e., the potential formation of other complexes containing Ag atoms is neglected, and (iii) that the defect concentrations are in local equilibrium. The deviation from stoichiometry $\Delta=\left[\mathrm{Cd}_{\mathrm{i}}\right]-\left[V_{\mathrm{Cd}}\right]$ of the crystal then is controlled by the difference of the concentrations of the intrinsic defects of the $\mathrm{Cd}$ sublattice. The incorporation of $\mathrm{Ag}$ in CdTe is determined by the defect reactions described by Eqs. (1) and (2) which represent the wellknown "kickout" and "dissociative" mechanisms, respectively [1]. Supplementing these equations by the annihilation reaction between the intrinsic defects $\mathrm{Cd}_{\mathrm{i}}+$ $V_{\mathrm{Cd}} \rightleftharpoons 0$ it can be shown that the ratio of the concentrations of interstitially and substitutionally incorporated $\mathrm{Ag}$ atoms is proportional to the square root of the ratio of the concentrations of the intrinsic defects of the $\mathrm{Cd}$ sublattice

$$
\left[\mathrm{Ag}_{\mathrm{i}}\right] /\left[\mathrm{Ag}_{\mathrm{s}}\right]=C\left(\left[\mathrm{Cd}_{\mathrm{i}}\right] /\left[V_{\mathrm{Cd}}\right]\right)^{1 / 2}
$$

whereby $C$ is a function of the temperature dependent equilibrium constants determining the reactions described in Eqs. (1) and (2). If the concentration of $\mathrm{Cd}_{\mathrm{i}}$ defects is increased, Eq. (3) shows that the concentration of $\mathrm{Ag}_{\mathrm{s}}$ atoms decreases in favor of $\mathrm{Ag}_{\mathrm{i}}$ atoms which diffuse into regions of higher $V_{\mathrm{Cd}}$ concentration. Taking into account that the measured diffusion profiles essentially correspond to $\mathrm{Ag}_{\mathrm{s}}$ atoms, this behavior explains the experimental diffusion data in a qualitative way: The Ag depleted layers observed correspond to regions of the crystals where the ratio $\left[\mathrm{Cd}_{\mathrm{i}}\right] /\left[V_{\mathrm{Cd}}\right]$ is high, e.g., caused by the respective thermal treatment under $\mathrm{Cd}$ vapor. Accordingly, the regions of high $\mathrm{Ag}$ concentrations correspond to a low ratio $\left[\mathrm{Cd}_{\mathrm{i}}\right] /\left[V_{\mathrm{Cd}}\right]$, which, e.g., is caused by the respective treatment under Te vapor; the ratio $\left[\mathrm{Cd}_{\mathrm{i}}\right] /\left[V_{\mathrm{Cd}}\right]$ obviously is also low in the CdTe crystals as delivered.

Since the ratio $\left[\mathrm{Cd}_{\mathrm{i}}\right] /\left[V_{\mathrm{Cd}}\right]$ directly corresponds to the deviation from stoichiometry, the different $\mathrm{Ag}$ profiles reflect the variation of the deviation from stoichiometry of the CdTe crystal as a function of depth and time at the respective temperatures and external vapor pressures of $\mathrm{Cd}$ and $\mathrm{Te}$. Consequently, the diffusion profiles of $\mathrm{Ag}$ should be the result of the so called chemical selfdiffusion in CdTe [10], which describes, using a diffusion coefficient $D(\Delta)$, the variation of the deviation from stoichiometry as a function of depth and time due to annealing under the respective external vapor pressures. Using the chemical self-diffusion coefficient $D(\Delta)=$ $5 \exp \left(-1.12 \mathrm{eV} / k_{B} T\right) \mathrm{cm}^{2} / \mathrm{s}$ reported for CdTe, which was determined using high temperature electrical conductivity measurements [11], a value of $4.4 \times 10^{-7} \mathrm{~cm}^{2} / \mathrm{s}$ at $800 \mathrm{~K}$ is obtained, yielding a diffusion length of $400 \mu \mathrm{m}$ after $60 \mathrm{~min}$ diffusion time. This value is in good agreement with the width of the depletion layers of the $\mathrm{Ag}$ profile shown in Fig. 2(a).

Using the model outlined above with the assumption that $\mathrm{Ag}_{\mathrm{i}}$ defects are highly mobile in agreement with the literature [9] and the mobility of $\mathrm{Ag}_{\mathrm{s}}$ can be neglected, a consistent fit to the different diffusion profiles shown in Fig. 2 is obtained (see solid lines). For these calculations a system of four coupled partial differential equations for the four defects $\mathrm{Ag}_{\mathrm{s}}, \mathrm{Ag}_{\mathrm{i}}, \mathrm{Cd}_{\mathrm{i}}, V_{\mathrm{Cd}}$ is used and the experimental data are treated in the limit of local equilibrium, as described in more detail in Ref. [7]. Obviously, the quite different experimental peak-shaped and U-shaped profiles can be described quantitatively, whereby the Ag profiles at low concentration levels seem to be reproduced more precisely. As expected, it turns out that the diffusivity of $V_{\mathrm{Cd}}$ is much smaller than that of $\mathrm{Cd}_{\mathrm{i}}$, yielding as upper limits $5 \times 10^{-9}$ and $1.75 \times 10^{-6} \mathrm{~cm}^{2} / \mathrm{s}$, respectively. For the diffusivity of the $\mathrm{Ag}_{\mathrm{i}}$ defects a lower limit of $D\left(\mathrm{Ag}_{\mathrm{i}}\right)=$ $2.0 \times 10^{-5} \mathrm{~cm}^{2} / \mathrm{s}$ is obtained in the case of diffusion under $\mathrm{Cd}$ pressure. In the case of diffusion under $\mathrm{Te}$ pressure, the asymmetric profile in the interior of the crystal yields $D\left(\mathrm{Ag}_{\mathrm{i}}\right)=8.4 \times 10^{-6} \mathrm{~cm}^{2} / \mathrm{s}$. The actual diffusivity of the $\mathrm{Ag}_{\mathrm{i}}$ defects in the framework of the present model depends on the external conditions during diffusion 
but it is always higher than the diffusivity of the $\mathrm{Cd}_{\mathrm{i}}$ defects. The good agreement with the experimental data shows that the basic idea of the model is correct. The enhanced surface concentration of $\mathrm{Ag}$ observable in Figs. 1(c), 2(c), 2(d), and 3 might indicate segregation effects or the formation of a different phase at the surface like, e.g., $\mathrm{Ag}_{2}$ Te.

For comparison, the diffusion data of Wartlick et al. [9] extrapolated to higher temperatures yield $D(\mathrm{Ag})=2.7 \times$ $10^{-7} \mathrm{~cm}^{2} / \mathrm{s}$ at $800 \mathrm{~K}$. This value, which is in between $D\left(\mathrm{Cd}_{\mathrm{i}}\right)$ and $D\left(V_{\mathrm{Cd}}\right)$ mentioned above, seems to be plausible since the effective diffusion of $\mathrm{Ag}_{\mathrm{i}}$ defects via simultaneously operating kickout and dissociative mechanisms is essentially determined by the diffusion of the intrinsic defects $\mathrm{Cd}_{\mathrm{i}}$ and $V_{\mathrm{Cd}}$; on the other hand, these intrinsic defects determine the chemical self-diffusion coefficient yielding a value of $D(\Delta)=4.4 \times 10^{-7} \mathrm{~cm}^{2} / \mathrm{s}$ at $800 \mathrm{~K}$ which is close to the extrapolated one. A more detailed discussion will be found in a separate publication [12]. It is also interesting to note that the present results are supported by an earlier publication by Schaake et al. [13] who investigated a new gettering mechanism for fast diffusing impurity atoms in $(\mathrm{Hg}, \mathrm{Cd}) \mathrm{Te}$. Their model along with the measured concentration profile of ${ }^{110 \mathrm{~m}} \mathrm{Ag}$ in $\mathrm{Hg}_{0.8} \mathrm{Cd}_{0.2} \mathrm{Te}$ fit to the conclusions of the present work extremely well. Finally, the wide range of diffusion coefficients reported for $\mathrm{Ag}$ in CdTe in the literature become understandable if the different experimental conditions are taken into account. Especially, the low diffusion coefficient measured in heavily donor doped CdTe crystals [14] seems to be plausible taking into account that donors are frequently compensated by the formation of $V_{\mathrm{Cd}}$ defects. The analysis of the diffusion data in Figs. 2(b) and 2(d) showed that a high $V_{\mathrm{Cd}}$ concentration drastically reduces the diffusivity of $\mathrm{Ag}$ as is reflected by the large fraction of the $\mathrm{Ag}$ atoms remaining within a layer of about $20 \mu \mathrm{m}$ below the surface. Also the results for the undoped CdTe obtained by Lyubomirsky et al. [15] and the p-type CdTe investigated by Kovalets et al. [16] may be well understandable in view of the presence of a high and a low concentration of $V_{\mathrm{Cd}}$ vacancies, respectively.

In conclusion, the presented data show that the diffusion profile of $\mathrm{Ag}$ in CdTe can be manipulated by varying the deviation from stoichiometry of the binary crystal with the help of the external vapor phase. The formations of the anomalous, peak-shaped diffusion profiles and their transitions to U-shaped profiles are well understood in terms of chemical self-diffusion. Thus, with the help of impurity diffusion profiles reflecting the distribution of intrinsic defects a new access to chemical self-diffusion might be obtainable. In order to get an improved quantitative description of the experimental data, it may be necessary to take into account the charge states of the participating defects and the potential influence of segregation effects. The diffusion profiles presented here should also be ob- servable in other compound semiconductors if the respective impurities diffuse interstitially and are more mobile than the corresponding self-interstitial defects. Indeed, similar diffusion profiles were already observed for $\mathrm{Cu}$ in $\mathrm{CdTe}$ and for $\mathrm{Ag}$ in $\mathrm{ZnTe}$ [12]. In addition, it is shown in $\mathrm{CdTe}$ that interstitial extrinsic defects, such as $\mathrm{Cu}$ atoms [see Fig. 1(a)] and Au atoms [12], affect the concentration profiles of $\mathrm{Ag}$ in a similar way like interstitial intrinsic defects. Finally, it should be noted that these defect interactions might be useful for gettering of unwanted impurities in semiconductors or, in general, for shaping of concentration profiles of impurity atoms in compound semiconductors.

The experimental help by V. Ostheimer and N.A. Stolwijk as well as the fruitful discussions with R. Grill, E. Belas, and M. Deicher are gratefully acknowledged. The financial support by the DFG under contract no. Wi715/-1 and by the BMBF under contract no. $05 \mathrm{KK} 1 \mathrm{TSB} / 5$ is gratefully acknowledged.

[1] U. M. Gösele and Teh Y. Tan, in Electronic Structure and Properties of Semiconductors, edited by W. Schröter, Materials Science and Technology Vol. 4, p. 197 (VCH, Weinheim, 1991)

[2] The only exception known to us is Pt in Si [H. Zimmermann and H. Ryssel, Appl. Phys. Lett. 59, 1209 (1991)].

[3] B. L. Sharma, Defect Diffus. Forum 64/65, 77 (1989).

[4] D. Shaw in Widegap II-VI Compounds for Optoelectonic Applications, Edited by H. E. Ruda (Chapman and Hall, London, 1992) Chap. 10.

[5] H. Wolf, M. Deicher, V. Ostheimer, A. Rodriguez Schachtrup, N.A. Stolwijk, Th. Wichert, and the ISOLDE Collaboration, Physica (Amsterdam) 308-310B, 963 (2001).

[6] H. Wolf , F. Wagner, and T. Wichert, Physica (Amsterdam) 340B, 275 (2003).

[7] H. Wolf, F. Wagner, Th. Wichert, and the ISLODE collaboration, Defect Diffus. Forum 237-240, 491 (2005).

[8] H. Mehrer, Phys. Status Solidi (a) 104, 247 (1987).

[9] B. O. Wartlick, C. Blanchard, and J. F. Barbot, Mater. Sci. Eng. B71, 254 (2000).

[10] R. L. Guldi, J. N. Walpole, and R.H. Rediker, J. Appl. Phys. 44, 4896 (1973).

[11] K. Zanio, J. Appl. Phys. 41, 1935 (1970).

[12] F. Wagner et al., (to be published).

[13] H.F. Schaake, J.H. Tregilgas, J.D. Beck, and M. A. Kinch, Solid State Commun. 50, 133 (1984); H. F. Schaake, J.H. Tregilgas, J. D. Beck, M. A. Kinch, and B. E. Gnade, J. Vac. Sci. Technol. A 3, 143 (1985).

[14] I. Lyubomirsky, V. Lyakhovitskaya, R. Triboulet, and D. Cahen, J. Electron. Mater. 26, 97 (1997).

[15] I. Lyubomirsky, M. K. Rabinal, and C. Cahen, J. Appl. Phys. 81, 6684 (1997).

[16] M. A. Kovalets, N. I. Kuchman, E. S. Nikonyuk, I.P. Chiokan, and M.M. Shvydka, Fizika i Khimiya Obrabotki Materialov 21, 125 (1987). 